\title{
Plasmodium yoelii infection inhibits murine leukaemia WEHI-3 cell proliferation in vivo by promoting immune responses
}

\author{
Zhen-Zhen Tong ', Zheng-Ming Fang', Qi Zhang ${ }^{1}$, Yun Zhan'1, Yue Zhang ${ }^{2}$, Wan-Fang Jiang ${ }^{1}$, Xiao Hou', \\ Yong-Long $\mathrm{Li}^{1}$ and Ting Wang ${ }^{1 *}$
}

\begin{abstract}
Background: Leukaemia is a malignant leukocyte disorder with a high fatality rate, and current treatments for this disease are unsatisfactory. Therefore, new therapeutic strategies for leukaemia must be developed. Malaria parasite infection has been shown to be effective at combating certain neoplasms in animal experiments. This study is to demonstrate the anti-leukaemia activity of malaria parasite Plasmodium yoelii (P. yoelii) infection,.

Methods: In this study, the proportion of CD3, CD19, CD11b and Mac-3 cells was analysed by flow cytometry; the levels of IFN- $\gamma$ and TNF-a in individual serum samples were measured by enzyme-linked immunosorbent assay, and the phagocytic activity of macrophages and natural killer (NK) cell activity were measured by flow cytometry.

Results: We found that $P$. yoelii infection significantly attenuated the growth of WEHI-3 cells in mice. In addition, tumor cell infiltration into the murine liver and spleen was markedly reduced. We also demonstrated that malaria parasite infection elicited anti-leukaemia activity by promoting immune responses, including increasing the surface markers of T cells (CD3) and B cells (CD19); decreasing the surface markers of monocytes (CD11b) and macrophages (Mac-3); inducing the secretion of IFN- $\gamma$ and TNF-a; and increasing NK cell and macrophage activity.

Conclusions: Malaria parasite infection significantly decreases the number of myeloblasts and inhibits neoplasm proliferation in mice. In addition, malaria parasite infection inhibits murine leukaemia by promoting immune responses.
\end{abstract}

Keywords: Leukaemia, Plasmodium yoelii, WEHI-3 cells, Anti-leukaemia

\section{Multilingual abstracts}

Please see Additional file 1 for translations of the abstract into the five official working languages of the United Nations.

\section{Background}

Leukemia comprises a group of malignant hematologic disorders. The disease incidence is approximately $15 / 100$ 000 individuals. In the United States and China, leukaemia accounts for the greatest proportion of childhood cancer cases $[1,2]$. Current treatments for the disease

\footnotetext{
* Correspondence: wangting139@hust.edu.cn

1Department of Parasitology, School of Basic Medicine, Tongji Medical College, Huazhong University of Science and Technology, Wuhan, Hubei, China

Full list of author information is available at the end of the article
}

remain unsatisfactory; therefore, new therapies must be established.

Recently, several researchers have reported an adverse relationship between parasitic infections and cancer. Parasitic infections, such as Trypanosoma cruzi [3], Toxoplasma gondii [4], Trichinella spiralis [5, 6], Toxocara canis [7], Acanthamoeba castellanii [8] and Plasmodium yoelii infections [9], have reportedly inhibited cancer growth in animal experiments. In addition, the adverse relationship between parasitic infections and cancer in humans has been demonstrated in epidemiological investigations of the prevalence of parasitic infections and cancer diseases [10]. In 1980, statistical data from the WHO indicated that the incidence of cancer was lowest in malaria-endemic areas [11].

(c) The Author(s). 2018 Open Access This article is distributed under the terms of the Creative Commons Attribution 4.0 International License (http://creativecommons.org/licenses/by/4.0/), which permits unrestricted use, distribution, and 
Although the mechanisms underlying the anticancer activity of some parasites are unclear, the mechanisms may be associated with antigens that are shared by parasites and cancer; these antigens may increase immune responses, which may nonspecifically induce anticancer activities [10]. In the present study, we examined the anti-leukaemia activity of $P$. yoelii infection in mice bearing WEHI-3 leukaemia cells and found that malaria parasite infection significantly attenuated WEHI-3 cell proliferation in these mice. We also demonstrated that $P$. yoelii infection induced anti-leukaemia activity by promoting immune responses.

\section{Methods}

\section{Mice and parasites}

We obtained 8- to 10-week-old female BALB/c mice from the Experimental Animal Center at Tongji Medical College (China; rodent license no. SYXK (e) 2010-0057). The animals were housed under specific-pathogen-free conditions in the animal facility and provided a sterile diet and autoclaved water. The animals were acclimated for 1 week prior to starting the experiment. All experimental procedures involving animals were approved by the Animal Research Ethics Committee of Tongji Medical College and performed in accordance with the institutional guidelines for the humane and ethical care of animals.

The $P$. yoelii 17XNL strain was obtained from Third Military Medical University in China.

\section{Murine WEHI-3 leukaemia cells}

The murine WEHI-3 myelomonocytic leukaemia cell line was obtained from the Cell Resource Center of the Institute of Basic Medical Sciences at the Chinese Academy of Medical Sciences. Cells were cultured in high-glucose DMEM containing 10\% FBS, 100 units/ml penicillin, $100 \mu \mathrm{g} / \mathrm{ml}$ streptomycin and $2 \mathrm{mmol} / \mathrm{L} \mathrm{L-glu-}$ tamine at $5 \% \mathrm{CO}_{2}$ and $37{ }^{\circ} \mathrm{C}$.

\section{Establishment of the murine leukaemia model and infection with the malaria parasite}

A murine leukaemia model was established as described by $\mathrm{He}$ and $\mathrm{Na}$ [12]. A total of $40 \mathrm{BALB} / \mathrm{C}$ mice were divided into four groups (10 animals per group). Group I (con) consisted of control mice; group II (Py) mice were intraperitoneally (i.p.) inoculated with $1 \times 10^{5} \mathrm{P}$. yoelii 17XNL-parasitized erythrocytes; group III (WEHI-3) mice were i.p. inoculated with $1 \times 10^{5}$ WEHI-3 cells; and group IV (WEHI-3 + Py) mice were i.p. inoculated with $1 \times 10^{5}$ WEHI-3 cells and then i.p. inoculated with $1 \times 10^{5} \mathrm{P}$. yoelii $17 \mathrm{XNL}$-parasitized erythrocytes 1 week later.

All mice were euthanized under anesthesia 2 weeks after inoculation with $P$. yoelii 17XNL. Each mouse was anesthetized by i.p. administration of $0.67 \%$ pentobarbital sodium at a dose of $100 \mu \mathrm{l} / 10 \mathrm{~g}$ body weight. No spontaneous deaths occurred before the mice were sacrificed. The blood, livers and spleens were collected from the mice, and bone marrow was flushed from the femurs of the sacrificed mice.

\section{Bone marrow smear and histopathological examination}

All bone marrow was flushed from the femurs of the sacrificed mice and smeared as described by Alabsi et al. [13]. Leukocyte classification based on cell morphology was performed by Wright's staining of the bone marrow smears, and the myeloblast percentages were determined by counting 500 nucleated bone marrow cells under a microscope.

Isolated spleen and liver samples were fixed in $4 \%$ formaldehyde, embedded in paraffin and sectioned at a thickness of $5 \mu \mathrm{m}$. The sections were stained with hematoxylin and eosin $(\mathrm{H} \& \mathrm{E})$ in accordance with the procedures described by Chung et al. [14] and were used for histopathological examination.

\section{Assay of natural killer (NK) cell activity}

Splenocytes were isolated from the fresh spleens of each mouse in all groups, and approximately $1 \times 10^{7}$ splenocytes were cultured in each well of 24-well culture plates. YAC-1 cells (NK target cells) obtained from the Laboratory of Cell Engineering of Tongji Medical College were stained according to the manufacturer's protocol (PKH67 Fluorescent Cell Linker Kits, Sigma-Aldrich Corp). Approximately $1 \times 10^{7}$ splenocytes from each mouse were mixed with labeled YAC-1 cells in the wells of a 96-well plate in an atmosphere containing $5 \% \mathrm{CO}_{2}$ at $37{ }^{\circ} \mathrm{C}$; the effector/target cell ratio was 50:1 or 100:1. After $12 \mathrm{~h}$ of incubation, NK cell activity was determined using a PI exclusion assay and flow cytometry (BD LSR II, USA) as described by Lin et al. [15].

\section{Assay of macrophage phagocytosis}

Macrophages were isolated from the peritoneal cavities of the control and experimental animals to investigate phagocytosis using a PHAGOTEST kit (Invitrogen V-6694 Vybrant ${ }^{\oplus}$, USA). Subsequently, for each mouse, a $0.5 \mathrm{ml}$ cell suspension (with $2 \times 10^{6}$ isolated cells $/ \mathrm{mL}$ ) in RPMI 1640 medium with $10 \%$ FBS was individually incubated with opsonized fluorescein isothiocyanate (FITC)-labeled E. coli $(0.5 \mathrm{ml})$ for $1.5 \mathrm{~h}$ at $37{ }^{\circ} \mathrm{C}$. In accordance with the manufacturer's instructions, ice-cold quenching solution $(100 \mu \mathrm{l})$ was added to stop the reaction. After completion of phagocytosis, the macrophages were analysed using a flow cytometer (BD LSR II, USA). 


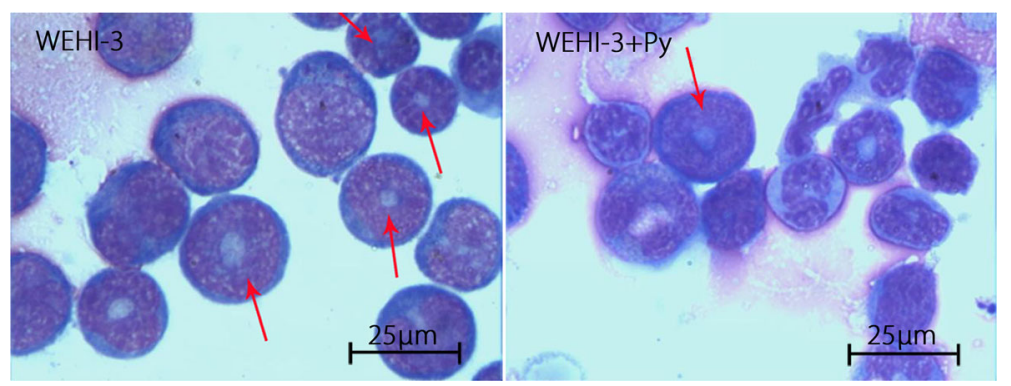

Fig. 1 Myeloblasts in bone marrow smears from mice in the WEHI-3 and WEHI-3 + P. yoelli (Py) groups. Mice were i.p. injected with WEHI-3 cells. After 3 weeks, the bone marrow was flushed from the femurs of the sacrificed mice and smeared. Leukocyte classification based on cell morphology was performed by Wright's staining of the bone marrow smears. The arrows $(\downarrow)$ indicate neoplasm cells (myeloblast), which contain large, irregular nuclei accompanied by prominent nucleoli and abundant light eosinophilic cytoplasm

Fluorescence data were collected for 10000 cells and analysed using Cellquest software.

\section{Assay of leukocyte surface markers}

For leukocyte collection, a blood sample from each mouse was immediately treated with ammonium chloride to lyse the red blood cells. The sample was then centrifuged at $1500 \mathrm{rpm}$ at $4{ }^{\circ} \mathrm{C}$ for $15 \mathrm{~min}$. After centrifugation, the supernatant was discarded, and the white blood cells were harvested. To measure cell surface markers of T cells (CD3), B cells (CD19), monocytes (CD11b) and macrophages (Mac-3), isolated white blood cells were stained with an anti-CD3-FITC antibody, an anti-CD19-phycoerythrin (PE) antibody, an anti-CD11b-FITC antibody and an anti-Mac-3-PE antibody (eBioscience, USA). The cell markers were

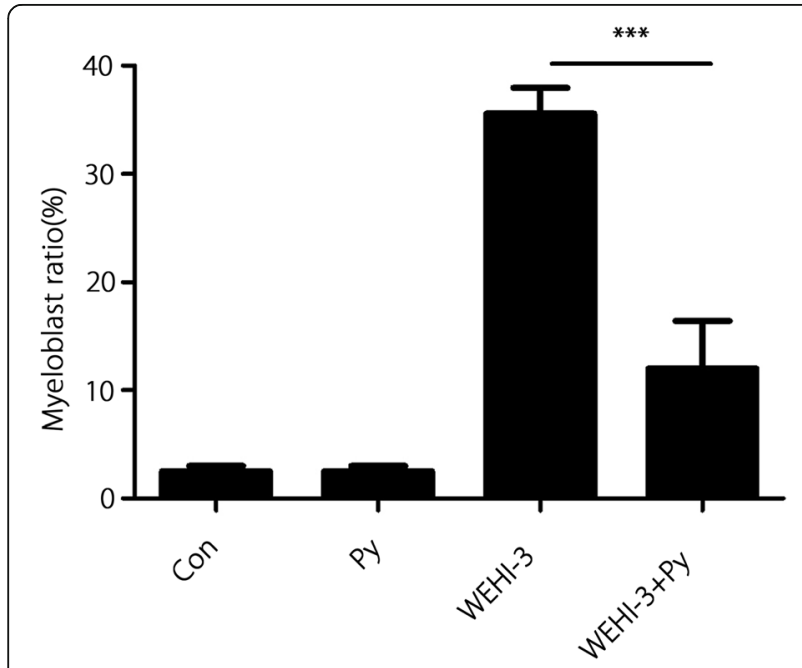

Fig. 2 Percentages of myeloblasts in bone marrow smears from mice of each group. The percentages of myeloblasts were determined by evaluating 500 nucleated bone marrow cells. The experiments were performed twice in duplicate, producing similar results; ${ }^{* *} P<0.001$ then analyzed by flow cytometry (BD LSR II, USA) as described by Lin et al. [15].

\section{Cytokine assay}

The levels of the Th1-type cytokines IFN- $\gamma$ and TNF- $\alpha$ were measured in each serum sample according to the instructions of the ELISA kit manufacturer (eBioscience, USA). The OD values of the reactions were read at $450 \mathrm{~nm}$ in an ELISA reader. The cytokine levels in the samples were calculated using standard curves constructed with known amounts of mouse recombinant IFN- $\gamma$ and TNF- $\alpha$ (eBioscience, USA), and the results are expressed in picograms per ml.

\section{Statistical analysis}

The data are expressed as the mean $\pm S D$, and differences between the control and experimental groups were analysed by one-way ANOVA. A value of $P<0.05$ was considered statistically significant.

\section{Results}

Bone marrow smear and histopathological examination Analysis of bone marrow smears showed that myeloblasts were present in the bone marrow of all mice (Fig. 1), but the percentage of myeloblasts in the mice inoculated with WEHI-3 cells was much higher than that in the mice of other groups $(P<0.001)$ (Fig. 2). After incubation with $P$. yoelii 17XNL, the percentage of myeloblasts in the mice inoculated with WEHI-3 cells decreased significantly (Fig. 2).

The histopathology results for liver and spleen tissues are shown in Figs. 3 and 4. In the WEHI-3 group, abundant neoplastic cells were found in liver and spleen tissues (Fig. 3), whereas the number of these cells were markedly reduced in the WEHI-3 + Py group $(P<0.01$ and $P<0.001$ compared with the liver (Fig. 4a) and 


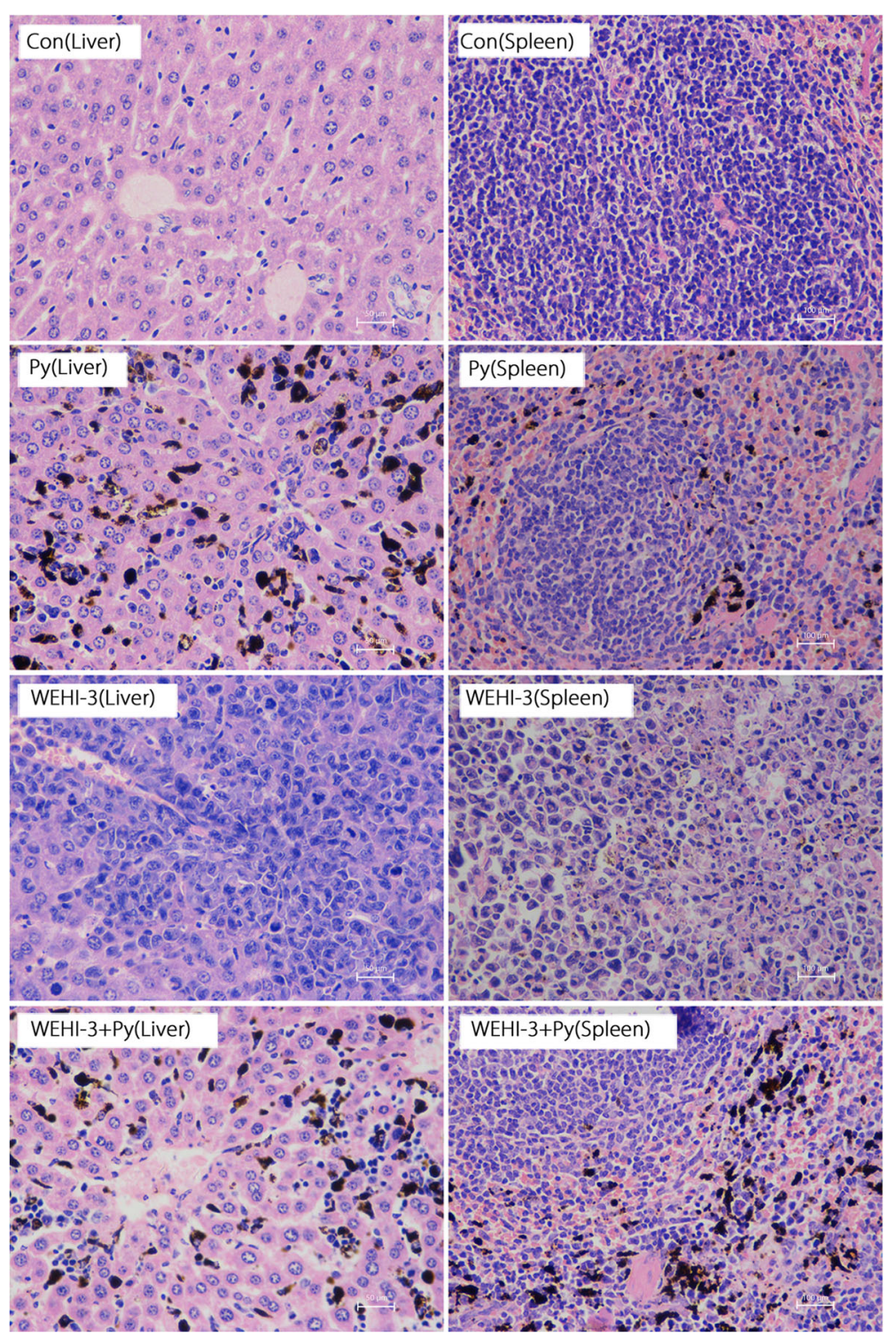

Fig. 3 Histopathological examination of liver and spleen tissues from each group. The isolated spleen and liver samples were fixed in $4 \%$ formaldehyde, embedded in paraffin and sectioned. The sections $(5 \mu \mathrm{m})$ were stained with H\&E and evaluated under a microscope at $400 \times$ magnification. The Con group shows normal structures and no infiltrated neoplasm cells in the liver and spleen. The P. yoelli (Py) group shows normal structures and no infiltrated neoplasm cells but shows deposition of malaria pigments in the liver and spleen. The WEHI-3 group shows abundant infiltrated neoplasm cells in the liver and spleen. The WEHI-3 + Py group shows markedly reduced neoplasm cell infiltration in the liver and spleen

spleen (Fig. 4b) tissues of the WEHI-3 group, respectively). The results indicated that malaria parasite infection could inhibit the development of leukaemia in mice.

\section{Effect of $P$. yoelii infection on NK cell activity}

The activity of NK cells isolated from the mouse spleens was measured, and the results showed that NK cell activity against YAC-1 target cells was significantly increased at target ratios of 50:1 and 100: 1 in the WEHI-3 + Py group compared with the
WEHI-3 group (Fig. 5), indicating that malaria parasite infection could increase NK cell cytotoxicity in WEHI-3 cell-bearing mice.

\section{Effect of $P$. yoelii infection on the phagocytic activity of macrophages}

Phagocytosis by macrophages isolated from the peritoneal cavity of mice in each group was evaluated using a flow cytometer, and the results shown in Fig. 6 indicate that malaria parasite infection promoted the phagocytic activity of macrophages in WEHI-3 cell-bearing mice. 

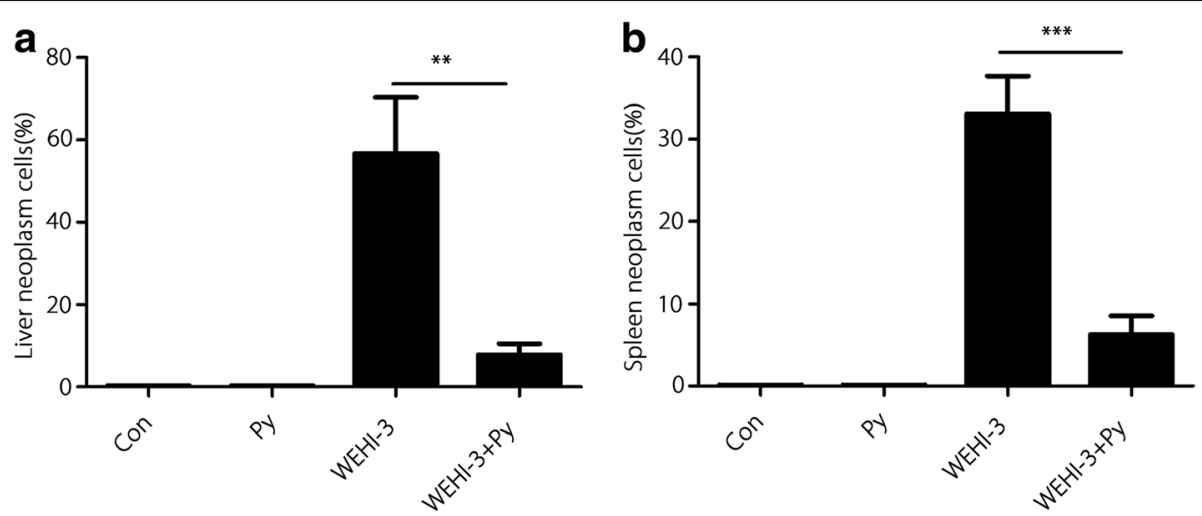

Fig. 4 Degree of neoplasm cell infiltration in the livers (a) and spleens (b) of mice. The mean proportion of neoplasm cells was calculated from five randomly selected fields by scanning the tissue sections at high power using an Olympus CX31 microscope (400 $\times$ magnification). The results are expressed as the mean $\pm S D$, and samples were obtained from 10 mice per group. ${ }^{* *} P<0.01$ and ${ }^{* * *} P<0.001$

\section{Surface markers of leukocytes in mice}

CD3, CD19, CD11b and Mac-3, the surface markers of leukocytes, were analysed in each group using flow cytometry. The data presented in Fig. 7 show that $P$. yoelii infection significantly increased the levels of CD3 (Fig. 7a) and CD19 (Fig. 7b) but decreased the levels of CD11b (Fig. 7c) and Mac-3 (Fig. 7d) compared with those levels in the WEHI-3 group. These results indicated that malaria parasite infection promoted differentiation of $\mathrm{T}$ and $\mathrm{B}$ cell precursors and inhibited differentiation of monocyte and macrophage precursors.

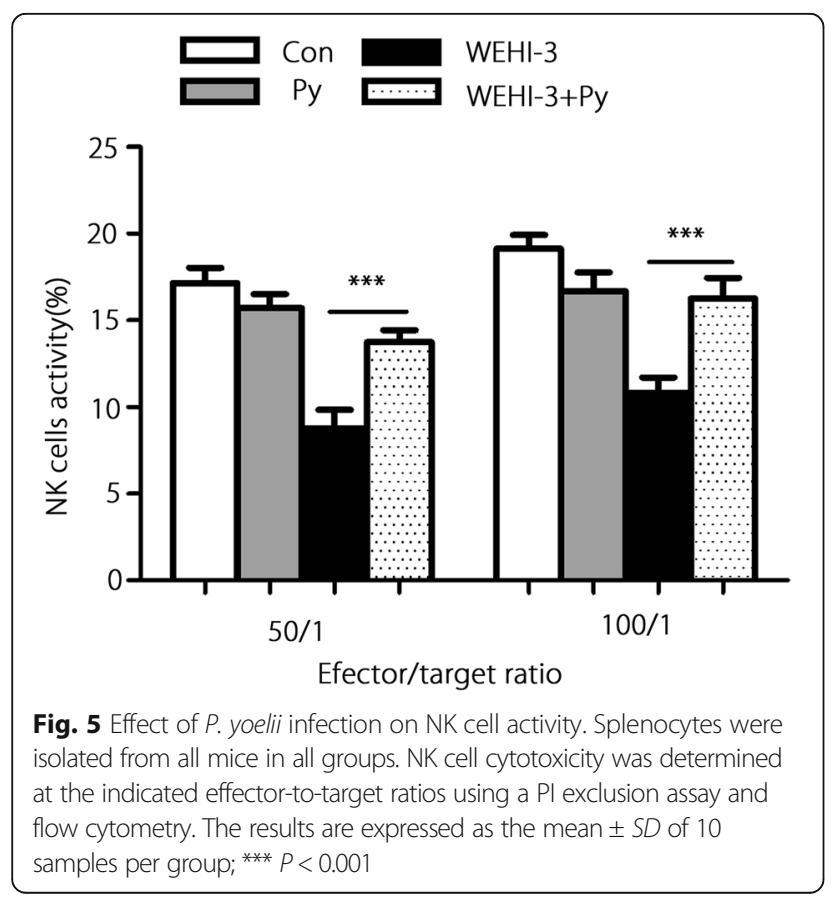

\section{Cytokine assays}

Changes in cytokine production in the sera from all groups are shown in Fig. $8 \mathrm{a}$ and b. P. yoelii infection (WEHI-3 + Py group) significantly increased the levels of the Th1-type cytokines IFN- $\gamma$ and TNF- $\alpha$ compared with those in the WEHI-3 group $(P<0.001)$.

\section{Discussion}

Leukaemia is an aggressive myeloid neoplasm characterized by arrest of myelopoietic cell maturation leading to myeloblast accumulation in the bone marrow and/or blood.

Leukaemia is diagnosed when myeloblasts constitute at least $20 \%$ of the nucleated cells in bone marrow or

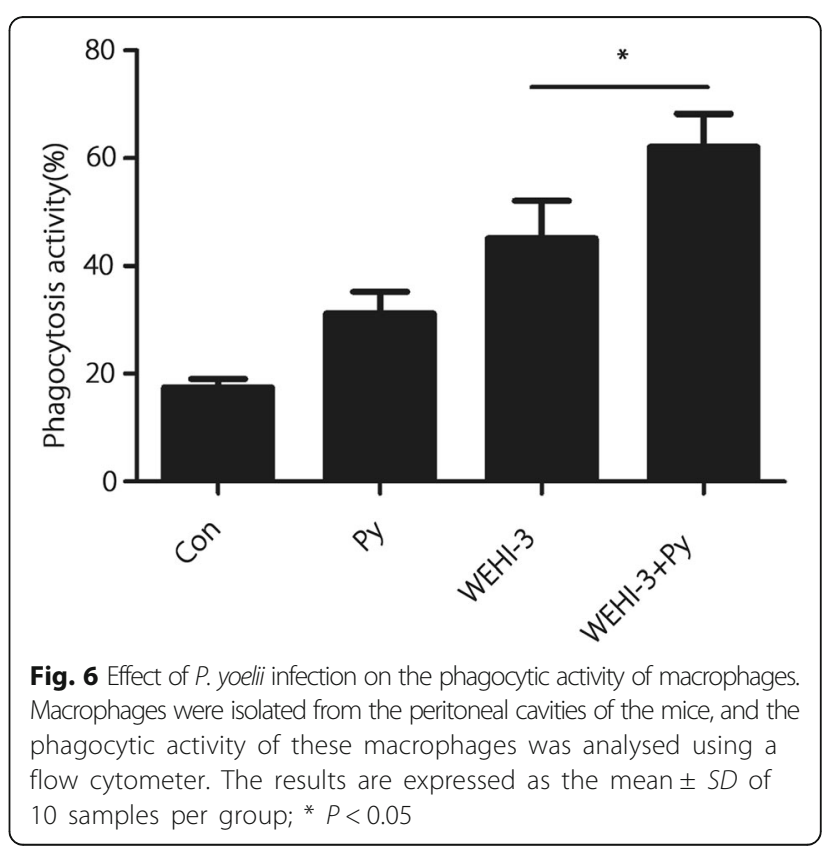



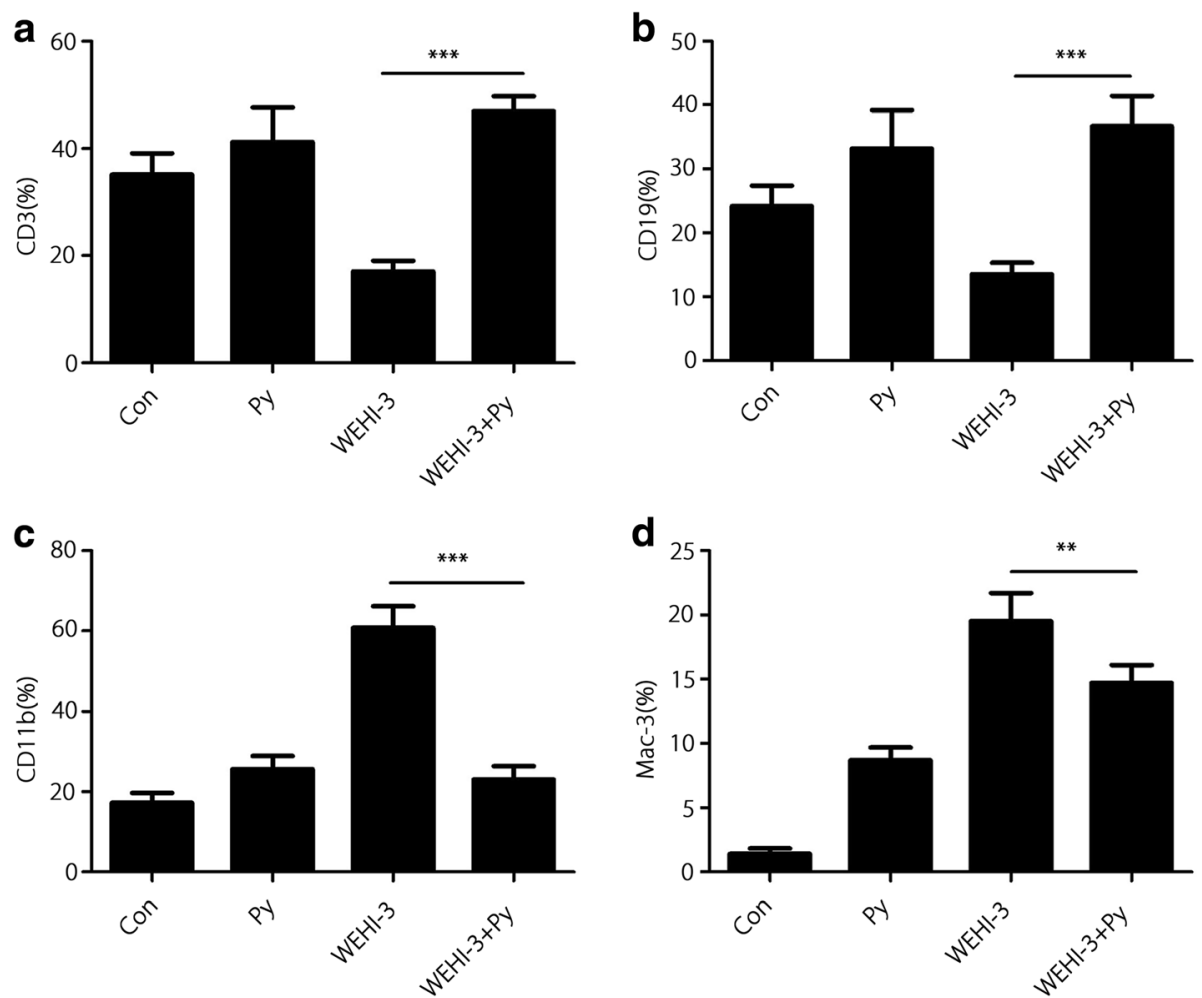

Fig. 7 Surface markers of leukocytes. White blood cells were collected from individual mice, and the specific cell surface markers CD3 (a), CD19 (b), CD11b (c) and Mac-3 (d) were analysed by flow cytometry. The results are expressed as the mean \pm SD, and samples were obtained from 10 mice per group. ${ }^{* *} P<0.01$ and ${ }^{* *} P<0.001$

blood [16]. Murine monomyelocytic leukaemia cells (WEHI-3) were originally derived from $\mathrm{BALB} / \mathrm{c}$ mice [17]. The major characteristics of the WEHI-3 leukaemia mice were elevated levels of peripheral monocytes and granulocytes with immature morphology, clearly enlarged and infiltrated spleens, and large numbers of undifferentiated cells in the bone marrow [12]. Therefore, the animal model fulfilled all the criteria of human myelomonocytic leukaemia [17].
In the present study, we demonstrated that myeloblasts accounted for $>30 \%$ of the non-erythroid cells in the bone marrow of mice inoculated with WEHI-3 cells, and we found neoplastic cells in the spleens and livers of these mice through histopathological examination. These changes indicated that the leukaemia mouse model was successfully established. After the WEHI-3 cell-bearing mice were inoculated with $P$. yoelii, the percentage of myeloblasts in the bone marrow was less than $20 \%$, and
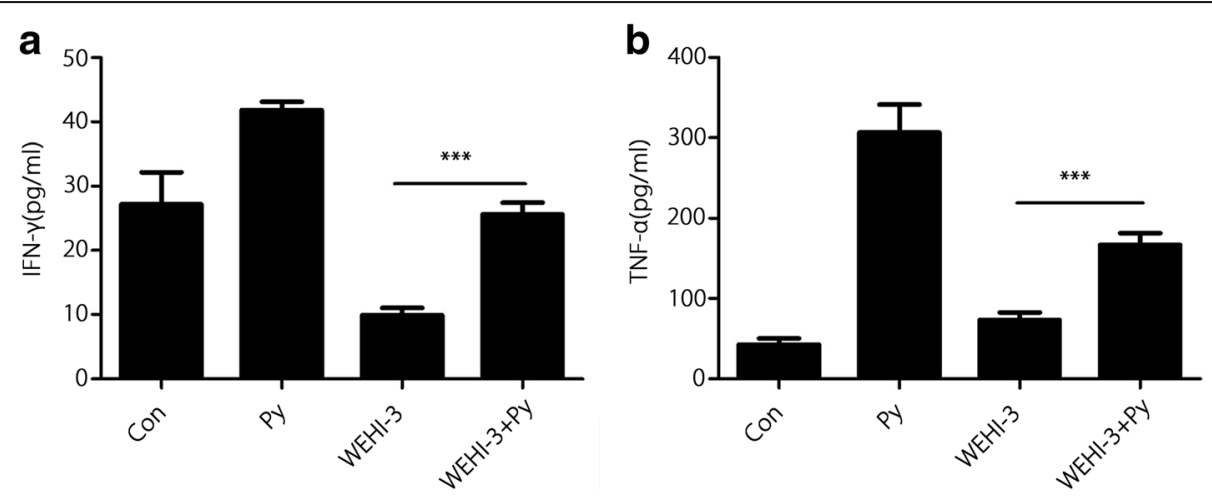

Fig. 8 . yoelii infection induced the production of IFN- $\gamma$ and TNF-a. The levels of IFN- $\gamma(\mathbf{a})$ and TNF- $a(\mathbf{b})$ in sera were measured by ELISA. The results are expressed as the mean $\pm S D$, and samples were obtained from 10 mice per group; ${ }^{* * *} P<0.001$ 
the number of neoplastic cells were notably decreased in the spleens and livers $(P<0.001$ and $P<0.01$, respectively). These results suggested that malaria parasite infection significantly decreased the number of myeloblasts and inhibited neoplasm proliferation in these mice.

Malaria is a parasitic disease caused by organisms in the Plasmodium genus, and an adverse relationship between parasitic infections and cancer has been reported [10]. Additionally, researchers have demonstrated that the human malaria parasite induces periodic high fever in the acute phase and that high fever can inhibit tumour growth [18]. However, the P. yoelii 17XNL strain does not induce fever in rodent malaria [19], and we did not observe fever in the animals in our study (data not shown). Therefore, the anti-leukaemia effect of $P$. yoelii $17 \mathrm{XNL}$ infection is not associated with hyperthermia.

The mechanisms of tumour resistance induced by parasites are not well understood, but various mechanisms may be involved, such as the presence of antigens common to tumours and parasites or concomitant immunity induced by living parasites such that tumours may be mistaken as parasites and subjected to an immune response [17]. In preliminary experiments, we used $P$. yoelii parasite lysates, but no anti-leukaemia activity was observed in the mice (data not shown), suggesting that the quantity of common antigens is very low in crude lysates of $P$. yoelii and that the virus must be extracted and purified. Additionally, this finding may also imply that the anti-leukaemia activity of $P$. yoelii infection is induced by living parasites. In fact, protective immunity to malaria is primarily a form of concomitant immunity and is effective only against residual populations of parasites [20]; therefore, lysates of $P$. yoelii are ineffective.

Malaria parasite infection can stimulate the host immune system, inducing polyclonal activation and massive proliferation and differentiation of lymphocytes with parasite-unrelated specificities $[21,22]$. In the present study, we demonstrated that $P$. yoelii infection could significantly decrease the percentages of CD11b and Mac-3 cells in the blood of mice inoculated with WEHI-3 cells, indicating that the differentiation of macrophage and monocyte precursors was inhibited. Meanwhile, $P$. yoelii infection increased the percentages of CD3 and CD19 cells in the mice, indicating that differentiation of the $\mathrm{T}$ and $\mathrm{B}$ cell precursors was enhanced. These results are consistent with those from other reports [23, 24]. Stimulation of T and B cell proliferation is believed to be efficacious in combating some cancers, including myeloid leukaemia [25-27]. We also found that malaria parasite infection could induce IFN- $\gamma$ and TNF$\alpha$ secretion, which may lead to improvements in the treatment of some cancers by inducing a massive influx of inflammatory cells as well as the production of Th1 cytokines [28-30]. P. yoelii infection enhanced T and B cell proliferation and increased the levels of the Th1 IFN- $\gamma$ and TNF- $\alpha$, suggesting that adaptive immunity is involved in the anti-leukaemia effect. Meanwhile, $P$. yoelii infection promoted NK cell activity and macrophage phagocytosis; these scavenger cells are widely believed to be an essential component of host immune defences against myeloid leukaemia [25, 31, 32], indicating that innate immunity is also involved in the anti-leukaemia effects from $P$. yoelii infection.

In the present study, we found that the percentage of macrophages was decreased (Fig. 7) but the activity of macrophages was increased (Fig. 6) in the P. yoelii-infected mice bearing WEHI-3 cells compared to the noninfected mice bearing WEHI-3 cells. These results seem to be contradictory, although similar results have been reported by Tsou [33]. The decreased percentage of Mac-3-expressing cells indicated that differentiation of macrophage precursors was inhibited after $P$. yoelii infection and that $P$. yoelii infection could inhibit murine leukaemia WEHI-3 cell proliferation. The increase in macrophage activity may have been induced by the parasite, as Plasmodium infection has been shown to significantly enhance the phagocytic activity of macrophages due to increased IFN- $\gamma$ expression [34, 35], and in the present study, IFN- $\gamma$ secretion was found to be increased in mice after $P$. yoelii infection.

\section{Conclusions}

Malaria parasite infection can enhance immune responses, including innate and adaptive anti-leukaemia responses, thereby significantly decreasing the number of myeloblasts and inhibiting neoplasm proliferation in a mouse model. The results suggest that the malaria parasite may represent a novel strategy or therapeutic vaccine vector for an immune-based therapy for leukaemia.

Although malaria parasite infection elicits an antileukaemia effect, the use of parasitic infections against leukaemia under clinical conditions is impossible. However, cancers and parasites may share common antigens, which may explain why certain parasites exhibit anticancer activity [10]. Therefore, the active antigens of malaria parasites for leukaemia immunotherapy are worthy of further study and identification.

\section{Additional files}

Additional file 1: Multilingual abstracts in the five official working languages of the United Nations. (PDF $492 \mathrm{~kb}$ )

\section{Abbreviations}

CD: Cluster of differentiation; ELISA: Enzyme-linked immunosorbent assay; FBS: Fetal bovine serum; FITC: Fluorescein isothiocyanate; H\&E: Hematoxylin and eosin; IFN-ү: Interferon-gamma; NK: Natural killer cells; OD: Optical density; PI: Propidium iodide; TNF-a: Tumor necrosis factor-alpha 


\section{Acknowledgments}

We thank Prof. Fu-Sheng Huang for kindly providing us with the $P$. yoelii 17XNL strain. This research was performed in compliance with the laws of China and the guidelines of the authors' respective institutions.

\section{Availability of data and materials}

Please contact the author for data requests.

\section{Authors' contributions}

ZZT designed the search strategy, contributed to the experiments, conducted the data analysis and data interpretation and wrote the manuscript. ZMF helped with the culture of Plasmodium yoelii. YZ helped with the culture of WEHI-3 leukemia cells. YZ and WFJ helped complete the experiment. QZ and $\mathrm{XH}$ helped with the data analysis. YLL and TW played critical roles in the supervision of the project, including the study design, experimental operation data analysis and manuscript writing. The manuscript was approved by all authors.

\section{Ethics approval and consent to participate}

Ethical approval was obtained from the Ethical Review Committee (ERC) at Tongji Medical College of the Huazhong University of Science and Technology.

\section{Consent for publication}

All authors have provided their consent for publication.

\section{Competing interests}

The authors declare that they have no competing interests.

\section{Author details}

'Department of Parasitology, School of Basic Medicine, Tongji Medical College, Huazhong University of Science and Technology, Wuhan, Hubei, China. ${ }^{2}$ Department of Immunology, School of Basic Medicine, Tongji Medical College, Huazhong University of Science and Technology, Wuhan, Hubei, China.

Received: 16 May 2017 Accepted: 25 April 2018

Published online: 16 May 2018

\section{References}

1. Han J, Liu J, Zheng X, Ma Y, Xiao Q, Ding Y. Caring in nursing: investigating the meaning of caring from the perspective of Chinese children living with leukemia. Int J Nurs Sci. 2014;1:34-41.

2. Landrigan PJ. Childhood leukemias. N Engl J Med. 1995;333:1286.

3. Mel'nikov VG, Fierro Velasko FH, Dobrovinskaya OR. Suppression of growth and metastasizing of T-cell lymphoma in mice infected with American trypanosomiasis at different stages of experimental infection. Bull Exp Biol Med. 2004;137:475-8.

4. Pyo KH, Jung BK, Chai JY, Shin EH. Suppressed CD31 expression in sarcoma180 tumors after injection with Toxoplasma gondii lysate antigen in BALB/C mice. Korean J Parasitol. 2010;48:171-4.

5. Duan L, Li J, Cheng B, Lv Q, Gong PT, Su LB, et al. Identification of a novel gene product expressed by trichinella spiralis that binds antiserum to Sp2/0 myeloma cells. Vet Parasitol. 2013;194:183-5.

6. Wang XL, Fu BQ, Yang SJ, Wu XP, Cui GZ, Liu MF, et al. Trichinella spiralis-a potential anti-tumor agent. Vet Parasitol. 2009:159:249-52.

7. Darani HY, Shirzad H, Mansoori F, Zabardast N, Mahmoodzadeh M. Effects of Toxoplasma gondii and toxocara canis antigens on WEHI-164 fibrosarcoma growth in a mouse model. Korean J Parasitol. 2009:47:175-7.

8. Pidherney MS, Alizadeh H, Stewart GL, McCulley JP, Niederkorn JY. In vitro and in vivo tumoricidal properties of a pathogenic/free-living amoeba. Cancer Lett. 1993;72:91-8.

9. Chen $L, H e$ Z, Qin L, Li Q, Shi X, Zhao S, et al. Antitumor effect of malaria parasite infection in amurine Lewis lung cancer model through induction of innate and adaptive immunity. PLoS One. 2011;6:e24407.

10. Warner NL, Moore MA, Metcalf D. A transplantable myelomonocytic leukemiain BALB-c mice: cytology, karyotype, and muramidase content. J Natl Cancer Inst. 1969;43:963-82.

11. Silverberg E. Cancer statistics, 1983. CA Cancer J Clin. 1983:33:9-25.

12. He Q, Na X. The effects and mechanisms of a novel 2-aminosteroid on murine WEHI-3B leukemia cells in vitro and in vivo. Leuk Res. 2001;25: 455-61.
13. Alabsi AM, Ali R, Ideris A, Omar AR, Bejo MH, Yusoff K, et al. Anti-leukemic activity of Newcastle disease virus strains AF2240 and V4-UPM inmurine myelomonocytic leukemia in vivo. Leuk Res. 2012;36:634-45.

14. Chung JG, Yang JS, Huang LJ, Lee FY, Teng CM, Tsai SC, et al. Proteomic approach to studying the cytotoxicity of YC-1 on U937 leukemia cells and antileukemia activity in orthotopic model of leukemia mice. Proteomics. 2007;7:3305-17.

15. Lin SY, Sheen LY, Chiang BH, Yang JS, Pan JH, Chang YH, et al. Dietary effect of Antrodia camphorate extracts on immune responses in WEHI-3 leukemia BALB/c mice. Nutr Cancer. 2010;62:593-600.

16. Hasserjian RP. Acute myeloid leukemia: advances in diagnosis and classification. Int J Lab Hematol. 2013;35:358-66.

17. Darani HY, Yousefi M. Parasites and cancers: parasite antigens as possible targets for cancer immunotherapy. Future Oncol. 2012;8:1529-35.

18. Soares PI, Ferreira IM, Igreja RA, Novo CM, Borges JP. Application of hyperthermia for cancer treatment: recent patents review. Recent Pat Anticanc Drug Discov. 2012;7:64-73.

19. Day JF, Edman JD. The importance of disease induced changes in mammalian body temperature to mosquito blood feeding. Physiol. 1984;77: 447-52.

20. Robert LS. Foundations of parasitology. 8th ed. Boston: McGraw-Hill Company; 2009. p. 159.

21. Miyakoda M, Kimura D, Yuda M, Chinzei Y, Shibata Y, Honma K, et al. Malaria-specific and nonspecific activation of $\mathrm{CD}^{+}{ }^{+} \mathrm{T}$ cells during blood stage of Plasmodium berghei infection. J Immunol. 2008;181:1420-8.

22. Sardinha LR, D'Império Lima MR, Alvarez JM. Influence of the polyclonal activation induced by Plasmodium chabaudi on ongoing OVA-specific Band T-cell responses. Scand J Immunol. 2002;56:408-16.

23. Lu CC, Yang JS, Chiang JH, Hour MJ, Lin KL, Lin JJ, et al. Novel quinazolinone MJ-29 triggers endoplasmic reticulum stress and intrinsic apoptosis in murine leukemia WEHI-3 cells and inhibits leukemic mice. PLoS One. 2012;7:e36831.

24. Yu FS, Wu CC, Chen CT, Huang SP, Yang JS, Hsu YM, et al. Diallyl sulfide inhibits murine WEHI-3 leukemia cells in BALB/C mice in vitro and in vivo. Hum Exp Toxicol. 2009:28:785-90.

25. Lichtenegger FS, Schnorfeil FM, Hiddemann W, Subklewe M. Current strategies in immunotherapy for acute myeloid leukemia. Immunotherapy. 2013;5:63-78

26. Mizukami M, Hanagiri T, Shigematsu Y, Baba T, Fukuyama T, Nagata Y, et al. Effect of IgG produced by tumor-infiltrating B lymphocytes on lung tumor growth. Anticancer Res. 2006;26:1827-31.

27. Yasuda M, Takenoyama M, Obata Y, Sugaya M, So T, Hanagiri T, et al. Tumor-infiltrating B lymphocytes as a potential source of identifying tumor antigen in human lung cancer. Cancer Res. 2002:62:1751-6.

28. Bolkun L, Lemancewicz D, Jablonska E, Szumowska A, Bolkun-Skornicka U, et al. The impact of TNF superfamily molecules on overall survival in acute myeloid leukaemia: correlation with biological and clinical features. Ann Hematol. 2015:94:35-43.

29. Van den Heuvel MM, Burgers SA, van Zandwijk N. Immunotherapy in nonsmall-cell lung carcinoma: from inflammation to vaccination. Clin Lung Cancer. 2009;10:99-105.

30. Zhao Q, Tong L, He N, Feng G, Leng L, Sun W, et al. IFN- $\gamma$ mediates graftversus- breast cancer effects via enhancing cytotoxic T lymphocyte activity. Exp Ther Med. 2014:8:347-54.

31. Jeannin P, Duluc D, Delneste $Y$. IL-6 and leukemia-inhibitory factor are involved in the generation of tumor-associated macrophage: regulation by IFN- $\gamma$. Immunotherapy. 2011;3:23-6.

32. Miller JS, Soignier Y, Panoskaltsis-Mortari A, McNearney SA, Yun GH, Fautsch SK, et al. Successful adoptive transfer and in vivo expansion of human haploidentical NK cells in patients with cancer. Blood. 2005;105:3051-7.

33. Tsou MF, Peng CT, Shih MC, Yang JS, Lu CC, Chiang JH, et al. Benzyl isothiocyanate inhibits murine WEHI-3 leukemia cells in vitro and promotes phagocytosisin BALB/c mice in vivo. Leuk Res. 2009;33:1505-11.

34. Cui Y, Liu J, Wu Y, Ma SH, Cao YM. Research of suface molecules related molecules related macrophage phagocytosis from Plasmodium Yoelii infected DBA/2 mice. Acta Parasitol Med Entomol Sin. 2007:14:71-5.

35. Stevenson MM, Riley EM. Innate immunity to malaria. Nat Rev Immunol. 2004:4:169-80. 